\title{
Imaging of abdominal tumours: CT or MRI?
}

\author{
Øystein E. Olsen
}

Received: 11 November 2008 / Accepted: 12 November 2008 / Published online: 16 December 2008

(C) Springer-Verlag 2008

\begin{abstract}
The scope of this review is to discuss a theoretical approach to imaging policy, particularly in the perspective of radiation risk reduction. Decisions are ideally driven by empirical evidence about efficacy and risk, e.g., in classical hierarchical efficacy model. As a result of the paucity of empirical evidence (inevitable because of rapid technological development), a pragmatic model is needed. This should avoid overemphasis of factors that currently seem to hamper change, namely personal preference, local expertise, infrastructure, availability. Extrapolation of current general knowledge about CT and MRI demonstrates how a pragmatic approach can be applied in the real world with
\end{abstract}

Dr. Olsen has no relevant financial relationships or potential conflicts of interest related to the material to be presented.

Editor's note: The full text of Dr. Olsen's presentation is published in the proceedings of the 2008 ESPR meeting and can be found in Pediatr Radiol 2008; 38(Suppl 3):S452-S458. doi:10.1007/s00247008-0846-5

Ø. E. Olsen $(\bowtie)$

Radiology Department,

Great Ormond Street Hospital for Children NHS Trust,

Great Ormond Street,

London WC1N 3JH, UK

e-mail: olseno@gosh.nhs.uk intermediate goals such as (1) channeling patients from CT to MRI, and (2) reducing CT-delivered radiation. Increased utilisation of MRI in body imaging requires optimisation of scan protocols and equipment, and, being a very operatordependent modality, the active involvement of the radiologist. In CT dose reduction the main challenge is to benchmark the minimum radiation-dose requirement, and therefore the minimum required image quality that is diagnostically acceptable. As this will ultimately depend on pre-test likelihoods in institutional populations, it is difficult to issue general guidance, and local assessment remains a cornerstone in this effort. 